ATUALIZAÇ̃̃o

\title{
DERMATITE CERCARIANA
}

POR ESQUISTOSSOMATÍDEOS DE AVES:

É POSSÍVEL A OCORRÊNCIA DE CASOS NO BRASIL?

\section{Hudson Alves Pinto, Vitor Luís Tenório Mati e Alan Lane de Melo ${ }^{1}$}

\section{RESUMO}

A dermatite cercariana causada por larvas de esquistossomatídeos de aves tem sido considerada uma enfermidade emergente. Embora ainda não haja relato de casos no Brasil, algumas espécies destes trematódeos já foram encontradas em aves e os potenciais hospedeiros intermediários (moluscos) estão amplamente distribuídos pelo país. Em razão da dificuldade de diagnóstico clínico diferencial da dermatite causada por larvas de Schistosoma mansoni, é possível que outras dermatites cercarianas sejam negligenciadas em nosso meio. Neste trabalho são discutidos aspectos relacionados à identificação de áreas de risco, à pesquisa de formas larvares em moluscos aquáticos, às alterações clínicas verificadas em humanos, ao tratamento e à profilaxia desta enfermidade. Concluiu-se que são necessários esforços para o diagnóstico da dermatite cercariana por outros esquistossomatídeos e também que sejam desenvolvidos estudos visando ampliar o conhecimento sobre a ocorrência destes parasitos no Brasil.

DESCRITORES: Cercárias. Dermatites. Schistosomatidae. Moluscos. Prurido.

\section{ABSTRACT}

Swimmers' itch by avian schistosomes: is it possible in Brazil?

Cercarial dermatitis caused by avian schistosomes larvae is considered an emerging disease. Although no case has so far been reported in human beings in Brazil, these trematodes have already been found in birds, and the potential intermediate hosts (mollusks) are widely distributed in the country. Considering the difficulty of the clinical differential diagnosis with dermatitis caused by Schistosoma mansoni, it is possible that human cases of dermatitis by avian schistosomes are being underdiagnosed. Aspects regarding the identification of transmission foci, the survey of cercariae in aquatic mollusks, symptoms to be verified in clinical examination, treatment, and prophylaxis for

1 Laboratório de Taxonomia e Biologia de Invertebrados, Departamento de Parasitologia, Instituto de Ciências Biológicas, Universidade Federal de Minas Gerais. C.P. 486, 30123-970, Belo Horizonte, Brasil.

Endereço para correspondência: A. L. Melo. E-mail: aldemelo@icb.ufmg.br

Recebido para publicação em: 14/12/2011. Aceito em: 9/3/2012. 
the disease are discussed. Efforts to diagnose this dermatitis and to increase the knowledge about the occurrence of these parasites in Brazil are needed.

KEY WORDS: Cercariae. Dermatitis. Schistosomatidae. Mollusks. Itching.

\section{INTRODUÇÃO}

A dermatite cercariana (DC), também conhecida como "dermatite do nadador", "dermatite do pescador" ou "dermatite de banhista", é uma reação inflamatória cutânea à penetração de cercárias de trematódeos da família Schistosomatidae, na qual estão incluídas espécies que parasitam aves, mamíferos e, ocasionalmente, répteis. Embora a DC seja verificada em seres humanos durante a fase inicial da esquistossomose (doença produzida por algumas espécies de Schistosoma Weinland, 1858), manifestações cutâneas resultantes da penetração de larvas de trematódeos de aves são também relatadas. De fato, uma grande quantidade de casos de DC por larvas de esquistossomatídeos de aves tem sido diagnosticada principalmente na América do Norte e Europa, sendo considerada uma enfermidade emergente $(26,30,36)$.

O maior contato de seres humanos com coleções aquáticas, mudanças climáticas e eutrofização são fatores que podem alterar significativamente a biologia dos parasitos, dos moluscos transmissores e dos hospedeiros definitivos naturais, contribuindo para o aumento na frequência de casos humanos relatados nos últimos tempos $(30,36,59)$.

No Brasil, apesar de haver registros de algumas espécies de esquistossomatídeos de aves, o problema relacionado à DC causada por estes parasitos é negligenciado, tendo a presente atualização o objetivo de discutir aspectos relacionados a essa manifestação clínica, enfatizando a possibilidade de sua ocorrência no país.

\section{BREVE HISTÓRICO E DISTRIBUIÇÃO}

As manifestações clínicas resultantes da penetração cutânea de larvas de trematódeos em seres humanos são conhecidas desde meados do século XIX quando Yoshinao Fujii descreveu, em 1847, a fase inicial da doença de Katayama, infecção causada pelo Schistosoma japonicum Katsurada, 1904 (84). Lutz (54), ao elucidar o ciclo biológico do Schistosoma mansoni Sambon, 1907 no Brasil, verificou experimentalmente a ocorrência de $\mathrm{DC}$ em roedores expostos a larvas do parasito, além de registrar o relato de algumas pessoas sobre a ocorrência de prurido após banhos em lagoas possivelmente contaminadas por cercárias do parasito.

Quanto aos Schistosomatidae parasitos de aves, a primeira espécie conhecida - Ornithobilharzia canaliculata (Rudolphi, 1819) - foi descrita com base em material coletado por Johann Natterer em expedição científica realizada 
no Brasil no início do século XIX. Já as cercárias de esquistossomatídeos de aves foram primeiramente descritas por La Valette de St. George (45) como Cercaria ocellata encontrada em Lymnaea stagnalis (Limnaeus, 1758) na Europa em meados do século XIX $(12,81)$.

A penetração de cercárias de esquistosomatídeos de aves na pele humana foi relatada primeiramente nos EUA, em 1928, por Cort (14). Desde então, a região dos Grandes Lagos americanos tornou-se um foco potencial da enfermidade e vários estudos realizados ao longo da primeira metade do século $\mathrm{XX}$ forneceram informações relacionadas à epidemiologia e ao controle da DC (15). Posteriormente, foi verificada a ocorrência de casos em ambiente marinho, primeiramente na costa sul da Califórnia $(27,72)$ e na Ilha de Rodes $(81)$. Focos de DC foram ainda relatados nos estados do Novo México, Colorado e Califórnia (8, 9) e, principalmente no verão, na região centro-oeste dos $\operatorname{EUA}(52,92)$.

$\mathrm{Na}$ Europa, casos da enfermidade foram primeiramente registrados na Alemanha $(15,24)$, Inglaterra $(60,64)$ e França $(12,26)$. Desde então, centenas de casos foram relatados em vários outros países como Áustria (18), Dinamarca (46), Espanha (58), Holanda (76, 91), Islândia (40, 46, 77), Noruega (46, 78), Polônia (94), República Tcheca (38) e Suécia (46, 85).

$\mathrm{Na}$ África, Ásia e Oceania, os relatos de DC por parasitos de aves são escassos, existindo registros na África do Sul $(3,16)$, Austrália $(4,6,57)$, China (50, 53), Índia (1), Irã (22), Japão (33, 80), Nova Zelândia (56), Tailândia (44), dentre outros países. Na América do Sul, casos de DC por esquistosomatídeos de aves foram apenas relatados na Argentina $(11,82,83) \mathrm{e}$, mais recentemente, no Chile (90).

\section{ASPECTOS TAXONÔMICOS}

A família Schistosomatidae possui atualmente nove gêneros que parasitam o sistema circulatório de aves, dos quais quatro (assinalados com asterisco) já estão incriminados em casos humanos de DC: Allobilharzia Koláŕová, Rudolfová, Hampl \& Skirnísson, 2006; *Austrobilharzia Johnston, 1917; *Bilharziella Looss, 1899; Dendritobilharzia Skrabin \& Zakharow, 1920; *Gigantobilharzia Odhner, 1910; Jilinobilharzia Liu \& Bai, 1976; Macrobilharzia Travassos, 1922; Ornithobilharzia Odhner, 1912; *Trichobilharzia Skryabin \& Zakharow, 1920 (29, $36,86)$. As principais espécies associadas à ocorrência de DC pertencem ao gênero Trichobilharzia, que atualmente apresenta mais de 40 espécies descritas. Este é o grupo mais bem estudado relativamente a ciclo biológico, distribuição geográfica, interação parasito-hospedeiro e biologia molecular $(7,31)$.

No Brasil, os estudos relacionados aos Schistosomatidae de aves são escassos, pois apenas cinco espécies destes trematódeos foram registradas até o momento em sete espécies de aves (Quadro 1). Quanto à infecção de moluscos por larvas destes parasitos no país, Lutz (55) descreveu algumas cercárias de cauda bifurcada que possuem características compatíveis com larvas de Schistosomatidae. 
Quanto à identificação específica, apenas Dendritobilharzia pulverulenta (Braun, 1901) (=D. anatinarum Cheatum, 1941) já foi identificada em Biomphalaria straminea (Dunker, 1848) naturalmente infectada no estado de Minas Gerais (49). Embora ainda não existam relatos de infecção natural, a suscetibilidade experimental de Lymnaea columella Say, 1817 e Physa marmorata Guilding, 1828 à infecção por Trichobilharzia jequitibaensis Leite et al., 1978 já foi demonstrada (48). Para as demais espécies de Schistosomatidae de aves ocorrentes no país, os hospedeiros intermediários são ainda desconhecidos.

Considerando-se a ampla riqueza e a distribuição da avifauna e malacofauna brasileira, a diversidade de espécies de Schistosomatidae ocorrentes no país é, ao certo, subestimada, o que resulta, possivelmente, da escassez de levantamentos helmintológicos tanto nos hospedeiros vertebrados quanto nos moluscos hospedeiros intermediários.

Quadro 1. Espécies de Schistosomatidae reportadas em aves e moluscos no Brasil

\begin{tabular}{|c|c|c|c|}
\hline Espécie & Hospedeiro definitivo & Hospedeiro intermediário & Referências \\
\hline \multirow{3}{*}{$\begin{array}{l}\text { Ornithobilharzia } \\
\text { canaliculata } \\
\text { (Rudolphi, 1819) }\end{array}$} & $\begin{array}{l}\text { Larus dominicanus } \\
\text { (Lichtenstein, 1823) }\end{array}$ & Desconhecido & 89 \\
\hline & $\begin{array}{l}\text { Thalasseus maximus } \\
\text { (Boddaert, 1783) }\end{array}$ & & \\
\hline & Sterna sp. & & \\
\hline $\begin{array}{c}\text { Macrobilharzia } \\
\text { macrobilharzia } \\
\text { (Travassos, 1922) }\end{array}$ & $\begin{array}{l}\text { Anhinga anhinga } \\
\text { (Linnaeus, 1758) }\end{array}$ & Desconhecido & 35,87 \\
\hline $\begin{array}{l}\text { Schistosoma pirajai } \\
\text { (Travassos, 1932) }\end{array}$ & $\begin{array}{c}\text { Anas bahamensis } \\
\text { (Linnaeus, 1758) }\end{array}$ & Desconhecido & 88 \\
\hline \multirow{2}{*}{$\begin{array}{c}\text { Dendritobilharzia } \\
\text { pulverulenta } \\
\text { (Braun, 1901) }\end{array}$} & $\begin{array}{l}\text { Cairina moschata } \\
\text { (Linnaeus, 1758) }\end{array}$ & $\begin{array}{l}\text { Biomphalaria straminea } \\
\text { (Dunker, 1848) }\end{array}$ & 25,49 \\
\hline & $\begin{array}{l}\text { Netta peposaca } \\
\text { (Vieillot, 1816) }\end{array}$ & & 63,71 \\
\hline \multirow{2}{*}{$\begin{array}{c}\text { Trichobilharzia } \\
\text { jequitibaensis } \\
\text { (Leite et al., 1978) }\end{array}$} & \multirow{2}{*}{$\begin{array}{l}\text { Cairina moschata } \\
\text { (Linnaeus, 1758) }\end{array}$} & $\begin{array}{c}\text { Lymnaea columella } \\
\text { (Say, 1817) }\end{array}$ & 47,48 \\
\hline & & $\begin{array}{l}\text { Physa marmorata } \\
\text { (Guilding, 1828) }\end{array}$ & \\
\hline
\end{tabular}

\section{CICLO BIOLÓGICO}

Os esquistosomatídeos parasitos de aves envolvidos na ocorrência de DC apresentam, de maneira geral, ciclo biológico semelhante ao do S. mansoni. Os 
hospedeiros vertebrados naturais, principalmente aves aquáticas, eliminam ovos dos parasitos nas fezes. Destes eclodem miracídios que nadam ativamente à procura do molusco hospedeiro intermediário. Nos EUA e Europa, moluscos pulmonados das famílias Lymnaeidae (Lymnaea stagnalis (Linnaeus, 1758), Radix peregra (Müller, 1774)), Planorbidae (Gyraulus parvus (Say, 1817), Planorbis planorbis (Linnaeus, 1758)) e Physidae (Physa gyrina Say, 1821), nesta ordem de importância epidemiológica, já foram incriminados na transmissão de esquistosomatídeos de aves para o ser humano (30). Moluscos marinhos pertencentes às famílias Nassariidae (Ilyanassa obsoleta (Say 1822)), Littorinidae (Littorina keenae Rosewater, 1978) e Haminoeidae (Haminoea japonica Pilsbry, 1895) foram também incriminados em casos de DC na América do Norte (9).

Nos moluscos, os miracídios transformam-se em esporocistos e estes produzem, assexuadamente por poliembrionia, cercárias que emergem, na maioria das vezes, em grande número. Estas nadam ativamente e penetram a pele de um hospedeiro vertebrado. No hospedeiro habitual, a cercária se transforma em esquistossômulo, alcança a circulação sanguínea, realiza o ciclo pulmonar e se desenvolve em adultos (machos e fêmeas) no sistema sanguíneo de aves. A localização dos vermes adultos depende da espécie do parasito, tendo sido encontradas algumas nas veias mesentéricas, veias renais ou cavidade nasal.

As cercárias de esquistossomatídeos de aves apresentam uma baixa especificidade em relação aos hospedeiros vertebrados. Em hospedeiros não habituais, como mamíferos, incluindo os seres humanos, as larvas são capazes de penetrar a pele, entretanto são mortas pelos mecanismos de defesa destes hospedeiros, ocasionando a DC.

Estudos experimentais demonstraram que, em algumas espécies destes parasitos, um pequeno percentual das cercárias que penetram se transforma em esquistossômulo na pele de mamíferos, migra para os pulmões onde permanece por alguns dias, podendo ocasionar hemorragia pulmonar (68). O desenvolvimento posterior, entretanto, é inibido quando comparado com aves. Migração para o sistema nervoso central de roedores também foi relatada para algumas espécies, sendo verificados casos de paralisia nestes hospedeiros, principalmente em camundongos imunossuprimidos ou imunodeficientes (17, 28, 29, 43, 51). Embora alterações pulmonares e neuromotoras sejam relatadas experimentalmente, a possibilidade de ocorrência da fase pulmonar e as possíveis implicações em humanos são ainda desconhecidas (32).

\section{ASPECTOS CLÍNICOS, PATOLÓGICOS E IMUNITÁRIOS}

Denomina-se dermatite o processo inflamatório que acomete a pele, geralmente superficial e restrito à epiderme, mas que pode também atingir a derme e a hipoderme. Diferentes agentes patológicos podem induzir o surgimento de alterações cutâneas, até mesmo formas parasitárias (61). A penetração das 
cercárias de esquistossomatídeos através da pele provoca lesões e prurido, caracterizando um quadro agudo alérgico relacionado à liberação de histamina e outros mediadores inflamatórios.

A sintomatologia da DC normalmente é de curta duração, podendo se manifestar tanto por quadros clínicos assintomáticos, quanto por formas generalizadas, algumas vezes com acometimento sistêmico. As manifestações clínicas, que envolvem principalmente as áreas da pele diretamente expostas à água contendo cercárias, iniciam-se ainda na primeira hora após a exposição. São observadas desde lesões discretamente perceptíveis até aquelas mais graves com características urticariformes. Sumariamente, a DC caracteriza uma alteração na pele com lesões maculopapulares associadas a intenso prurido. A evolução das lesões segue um roteiro determinado. Habitualmente até 20 minutos após o contato com as larvas do parasito é relatada a ocorrência de prurido (primário). Máculas envoltas por áreas de eritema aparecem nos locais de penetração das cercárias, mas, na maioria das vezes, rapidamente desaparecem. Entretanto, há situações em que as máculas podem persistir por várias horas e evoluir para vesículas ou, mais frequentemente, pápulas enrijecidas, cujo aparecimento é acompanhado por prurido ainda mais intenso (secundário). Nestes casos, progressivamente regridem a partir de três a cinco dias após a penetração das larvas, sendo necessárias até duas semanas para que ocorra a remissão das lesões. Manchas pigmentadas então surgem e podem persistir por mais de um mês, sendo a hiperpigmentação da pele uma sequela possível. Edema e eritema difuso também podem ocorrer, mesmo que raramente, em substituição à reação macular, sobretudo em infecções mais intensas quando se verifica a confluência das lesões. Também pode haver linfangite, adenopatia regional, bem como predominância de lesões secundárias por infecção ou escoriação caracterizadas por exsudação e formação de crostas. Nestes casos, pústulas são também identificáveis. Além disso, a penetração de grande número de cercárias em seres humanos pode ser responsável por manifestações sistêmicas como febre, náuseas, diarreia, insônia relacionada ao prurido e eosinofilia $(14,15,31,61,66,79)$.

O tempo de duração e intensidade dos sintomas depende da sensibilidade do indivíduo afetado. Em geral, maiores frequência e intensidade da exposição às larvas dos trematódeos estão relacionadas diretamente a reações cutâneas mais graves (67).

Adermatite produzida por esquistossomatídeos de não humanos, sobretudo de aves, é, do ponto de vista clínico, classicamente reconhecida como uma condição mais significativa. Para alguns autores, as reações dérmicas à penetração de cercárias de Schistosoma que parasitam seres humanos são normalmente menos graves, não havendo a formação de pápulas $(5,15,23)$, embora a repetição de exposição a estas cercárias possa agravar o quadro dermatológico. Por outro lado, há também autores que afirmam que todos os gêneros de esquistossomatídeos induzem quadro clínico semelhante quando infectam humanos (31). De fato, Kolářová (36) discute, com base em dados não publicados, que não foram observadas diferenças na intensidade 
da reação cutânea entre indivíduos com DC adquirida na Europa (reconhecidamente infectados apenas por esquistossomatídeos de aves) e indivíduos infectados por cercárias de Schistosoma na África. No Brasil, embora pouca ênfase esteja sendo dada à descrição detalhada das lesões cutâneas, a DC tem sido associada apenas à esquistossomose mansoni aguda $(20,65,79)$.

Quanto às alterações histopatológicas, não se percebe grandes diferenças entre os fenômenos relacionados à penetração das cercárias de diferentes espécies de Schistosomatidae. Lesões do tegumento cutâneo são determinadas pelas cercárias destes parasitos que, por ação enzimática, produzem alterações da camada córnea e da barreira conjuntiva acelular. Biópsias de pele humana realizadas após a exposição às cercárias demonstraram larvas dentro de túneis na epiderme e um infiltrado inflamatório agudo discreto com predomínio de neutrófilos e eosinófilos, resultando em vasodilatação associada ao aumento da permeabilidade capilar. No fim do segundo dia de infecção, os túneis encontram-se normalmente sem cercárias ou com larvas em processo de histólise $(15,74)$.

O desenvolvimento de DC relaciona-se a repetidas infecções por cercárias que induzem uma resposta alérgica capaz de eliminar o parasito durante a penetração (36). Na primoinfecção, as manifestações clínicas são reconhecidamente mais brandas do que na reinfecção, tanto para larvas de parasitos de humanos quanto de aves. Por outro lado, se o hospedeiro foi previamente exposto a cercárias, reações de maior intensidade e duração são clinicamente verificadas (15, 31, 67), situação que remete à possibilidade de sensibilização cruzada entre antígenos das diferentes espécies de cercárias.

Embora a DC seja frequentemente descrita como uma reação de hipersensibilidade imediata, tem sido demonstrada também a participação de mecanismos relacionados à reação de hipersensibilidade tardia (42).

\section{IDENTIFICAÇÃO DE FOCOS DE TRANSMISSÃO}

Para a determinação de um potencial foco de DC, é necessária a realização de estudos malacológicos nas áreas suspeitas, especialmente em coleções aquáticas utilizadas para recreação. Os aspectos metodológicos relacionados à pesquisa de moluscos transmissores, sua identificação e avaliação da infecção por parasitos são, de modo geral, semelhantes à determinação das áreas de transmissão de $S$. mansoni. Resumidamente, após a coleta malacológica, os moluscos devem ser analisados individualmente, sendo colocados em recipientes contendo água e expostos à fotoestimulação artificial para posterior análise em estereomicroscópio. Uma ampla variedade de larvas de trematódeos pode ser encontrada em moluscos aquáticos e a identificação de larvas de esquistossomatídeos é necessária para a determinação de um potencial foco de DC. Para isso, as cercárias emergidas de moluscos infectados devem ser estudadas em microscópio de luz, a fresco, com auxílio de corantes vitais e após fixação, coloração e montagem de lâminas permanentes (62). 
Apesar de as larvas de Schistosomatidae de aves possuírem cauda bifurcada com furcas curtas (brevifurcada), não possuírem faringe e apresentarem os cecos curtos, tal como as cercárias de S. mansoni, elas diferem principalmente por apresentarem um par de ocelos pigmentados na região anterior do corpo. Outras furcocercárias encontradas em moluscos podem ser confundidas com cercárias de Schistosomatidae, não sendo capazes de penetrar a pele humana. Larvas de trematódeos da família Clinostomidae, parasitos do trato digestivo superior de aves, apresentam membrana natatória dorsal, possuem faringe e o acetábulo é rudimentar ou ausente. Cercárias de representantes da família Spirorchiidae, cujos adultos parasitam o sistema circulatório de quelônios, podem ser diferenciadas principalmente por apresentarem furcas contendo membrana natatória com espinhos, além de serem larvas relativamente maiores quando comparadas com cercárias de Schistosomatidae. Larvas do tipo estrigeocercária, formadas por representantes das famílias Cyathocotylidae, Diplostomidae e Strigeidae, diferem das larvas de Schistosomatidae principalmente por apresentarem as furcas longas (longifurcadas) e possuírem faringe.

A identificação específica das formas larvares encontradas em moluscos exige uma análise morfológica minuciosa das cercárias e a obtenção experimental de parasitos adultos em modelos experimentais. Aspectos metodológicos relacionados ao estudo laboratorial de esquistosomatídeos causadores de DC foram discutidos em detalhe por Kolářová et al. (2010) (37). Em alguns casos, a morfologia das larvas (número e disposição de glândulas de penetração, fórmula do sistema excretor e medidas) pode permitir a determinação do gênero do parasito. Ilustrações de diferentes cercárias de Schistosomatidae de aves descritas por vários autores podem ser consultadas em Combes (13) e Ostrowski de Nuñez (69, 70).

Nos últimos anos, estudos moleculares foram utilizados para a identificação de esquistossomatídeos de aves. A amplificação por reação em cadeia da polimerase (PCR) e o sequenciamento de marcadores moleculares, como a região espaçadora transcrita interna 1 e 2 do DNA ribossomal (ITS1 e ITS2 rDNA), a região codificadora dos genes $18 \mathrm{~S}-28 \mathrm{~S}$ do DNA ribossomal e do gene mitocondrial citocromo c oxidase subunidade I (COI), permitiram, além da identificação molecular, a obtenção de informações importantes sobre a filogenia destes parasitos $(2,7,9,10,19,34,75)$.

No Brasil, os estudos sobre larvas de esquistossomatídeos de aves potencialmente envolvidas na gênese de DC são escassos. Entretanto, cercárias destes parasitos (Figura 1) têm sido frequentemente observadas por nós em $B$. straminea coletadas durante estudos malacológicos realizados na represa da Pampulha, Belo Horizonte, Minas Gerais. Ensaios experimentais confirmaram a capacidade de penetração desta larva em pele de roedores, sendo observada macroscopicamente a ocorrência de hiperemia e vasodilatação (dados não publicados). Tentativas de elucidação do ciclo biológico e identificação do parasito estão sendo conduzidas. 


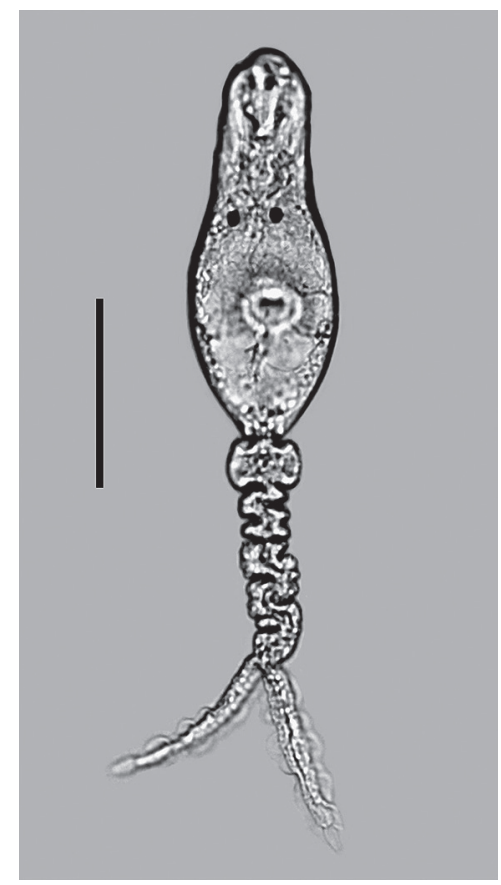

Figura 1. Cercária de Schistosomatidae de aves encontrada em Biomphalaria straminea na represa da Pampulha, Belo Horizonte, Minas Gerais. Escala $=100 \mu \mathrm{m}$.

\section{DIAGNÓSTICO, TRATAMENTO E PROFILAXIA}

O diagnóstico da DC por esquistossomatídeos de aves, por ser ainda eminentemente clínico, é baseado na história epidemiológica e nas características das lesões, sendo difícil de ser realizado em áreas endêmicas para esquistossomose. Embora sejam úteis as informações epidemiológicas, como o conhecimento dos cursos d'água frequentados pelos indivíduos, da fauna malacológica local e das cercárias encontradas em moluscos, as manifestações clínicas semelhantes comprometem o diagnóstico de certeza. No Brasil, muitos profissionais da saúde provavelmente não estejam atentos à possibilidade de ocorrência de DC por outros trematódeos. $\mathrm{O}$ diagnóstico diferencial entre a dermatite ocasionada por larvas de $S$. mansoni e a produzida por outros esquistossomatídeos ou pela penetração de larvas de nematódeos é difícil de ser realizado $(61,79)$. Testes sorológicos para diagnóstico diferencial de dermatite por S. mansoni e por outros esquistossomatídeos, como ELISA e reação de imunofluorescência indireta (RIFI), estão ainda em fase experimental $(39,41,61)$. 
Nas áreas onde não ocorrem casos de esquistossomose, o tratamento da DC é sintomático, dada sua condição autolimitada. Anti-histamínicos orais e corticosteroides tópicos são úteis para aliviar o desconforto. Ocasionalmente naqueles casos mais graves, um rápido ciclo de tratamento com corticosteroide oral pode ser necessário, também com antibióticos quando houver infecção bacteriana secundária (61). O diagnóstico diferencial entre DC por S. mansoni e outros esquistossomatídeos obviamente está relacionado à opção terapêutica, sobretudo ao se considerar que o tratamento com oxamniquina durante a fase aguda da esquistossomose mansoni pré-postural tem sido sugerido (21).

Em relação à profilaxia, algumas formulações cutâneas têm sido testadas na Europa. Niclosamida em baixas concentrações e uma formulação comercial contra águas-vivas (Safe-Sea) demonstraram-se capazes de inibir a penetração das cercárias de esquistossomatídeos de aves na pele de humanos (93). O diagnóstico e tratamento das aves naturalmente infectadas bem como a eliminação dos moluscos transmissores, apesar de difícil execução, foram sugeridos com uma forma de controle da enfermidade (31).

\section{PERSPECTIVAS E CONCLUSÃO}

A potencialidade de ocorrência de casos de DC por esquistossomatídeos de aves no Brasil é notória. Na década de 1930, Fülleborn já aventara a possibilidade de haver casos de DC por esquistossomatídeos de aves no Brasil em meio às dermatites descritas para $S$. mansoni, sobretudo ao se considerar a migração de aves oriundas de regiões endêmicas nos EUA para a região neotropical (15). Nesse sentido, é possível que o enfoque nas manifestações cutâneas por $S$. mansoni, embora necessário, tenha historicamente reduzido a atenção dos parasitologistas brasileiros às demais DC. Pessôa \& Martins (73) afirmaram que a ausência de casos de DC por trematódeos de aves em nosso país é resultante da falta de pesquisas, situação que pouco evoluiu até o presente.

O estudo de cercárias de esquistossomatídeos, sobretudo de aves, merece maior atenção no Brasil. Sugere-se um melhor monitoramento da malacofauna nacional com enfoque em cercárias potencialmente causadoras de DC e inquéritos destes parasitos nas aves ocorrentes em coleções aquáticas frequentadas por banhistas.

Considerando-se a presença de moluscos transmissores, os relatos prévios de algumas espécies destes parasitos no país, bem como a ocorrência mundial das DC por esquistossomatídeos de aves, inclusive em países vizinhos, é provável a ocorrência de casos no Brasil, os quais, dada a dificuldade de diagnóstico, podem estar sendo negligenciados.

\section{REFERÊNCIAS}

1. Agrawal MC, Gupta S, George J. Cercarial dermatitis in India. Bull World Health Organ 78: 278, 2000. 
2. Aldhoun JA, Podhorský M, Holická M, Horák P. Bird schistosomes in planorbid snails in the Czech Republic. Parasitol Int 61: 250-259, 2011.

3. Appleton CC. Schistosome dermatitis - an unrecognized problem in South Africa? S Afr Med J 65: 467-469, 1984.

4. Appleton CC, Lethbridge RC. Schistosome dermatitis in the Swan Estuary, Western Australia. Med J Aust Mar 10: 141-145, 1979.

5. Basch PF. Schistosomes. Development, Reproduction and Host Relations. Oxford University Press. Oxford, 1991.

6. Blair D, Copeman DB. Schistosome dermatitis in North Queensland. Med J Aust 1: 40, 1977.

7. Brant SV, Loker ES. Molecular systematics of the avian schistosome genus Trichobilharzia (Trematoda: Schistosomatidae) in North America. J Parasitol 95: 941-963, 2009a.

8. Brant SV, Loker ES. Schistosomes in the southwest United States and their potential for causing cercarial dermatitis or 'swimmer's itch'. J Helminthol 83: 191-198, $2009 \mathrm{~b}$.

9. Brant SV, Cohen AN, James D, Hui L, Hom A, Loker ES. Cercarial dermatitis transmitted by exotic marine snail. Emerg Infect Dis 16: 1357-1365, 2010.

10. Brant SV, Morgan JA, Mkoji GM, Snyder SD, Rajapakse RP, Loker ES. An approach to revealing blood fluke life cycles, taxonomy, and diversity: provision of key reference data including DNA sequence from single life cycle stages. J Parasitol 92: 77-88, 2006.

11. Bosq P, Szidat L, Soria MF. Dermatitis schistosomica por Cercaria chascomusi. Prensa Med Arg 42: 3500-3504, 1955.

12. Brumpt E. Prurit et dermatites produites chez les nageurs par des cercaires de mollusques d'eau douce. CR Acad Sci Paris 193: 253-255, 1931.

13. Combes C. Atlas Mondial des Cercaires. Mem Mus Natl Hist Nat, Ser A, Zool 115: 5-235, 1980.

14. Cort WW. Schistosome dermatitis in the United States (Michigan). J Am Med Assoc 90: 1027-1029, 1928.

15. Cort WW. Studies on schistosome dermatitis XI. Status of knowledge after more than twenty years. Am J Hyg 52: 251-307, 1950.

16. De Meillon B, Stoffberg N. Swimmer's itch in South Africa. S Afr Med J 28: 1062-1064, 1954.

17. Dvoř́ák J, Kolářová L, Trefil L. Trichobilharzia regenti, a pathogen of the avian and mammalian central nervous systems. Parasitology 119: 577-581, 1999.

18. Dvoř́k J, Sattmann H, Horák P, Konecny R. Bird schistosomes from freshwater snails in Austria, with some notes on current problems (Digenea, Schistosomatidae). Mitt Österr Ges Tropenmed Parasitol 21: 69-76, 1999.

19. Dvořák J, Vaňáčová Š, Hampl V, Flegr J, Horák P. Comparison of European Trichobilharzia species based on ITS1 and ITS2 sequences. Parasitology 124: 307-313, 2002.

20. Enk MJ, Amaral GL, Costa-Silva MF, Silveira-Lemos D, Teixeira-Carvalho A, Martins-Filho OA, Correa-Oliveira R, Gazinnelli G, Coelho PMZ, Massara CL. Rural tourism: a risk factor for schistosomiasis transmission in Brazil. Mem Inst Oswaldo Cruz 105: 537-540, 2010.

21. Enk MJ, Katz N, Coelho PMZ. A case of Schistosoma mansoni infection treated during the prepatent period. Nat Clin Pract Gastroenterol Hepatol 5: 112-115, 2008.

22. Farahnak A, Essalat M. A study on cercarial dermatitis in Khuzestan province, south western Iran. BMC Public Health 3: 35-38, 2003.

23. Faust EC, Jones CA, Hoffman WA. Studies on schistosomiasis mansoni in Puerto Rico. III. Biological studies. 2. The mammalian phase of the life cycle. Puerto Rico J Pub Health Trop 10: 133-189, 1934.

24. Fölster-Holst R, Disko R, Röwert J, Böckeler W, Kreiselmaier I, Christophers E. Cercarial dermatitis contracted via contact with an aquarium: case report and review. Br J Dermatol 145: 638-640, 2001.

25. Freitas MG, Costa HM. Dendritobilharzia anatinarum Cheatum, 1941 in the domestic duck, in Brazil (Trematoda, Schistosomatidae). Rev Bras Biol 32: 343-345, 1972.

26. Gentile L, Picot H, Bourdeau P, Bardet R, Kerjan A, Piriou M, Le Guennic A, Bayssade-Dufour C, Chabasse D, Mott KE. La dermatite cercarienne en Europe: un problème de sante publique nouveau? Bull World Health Organ 74: 159-163, 1996. 
27. Grodhaus G, Keh B. The marine, dermatitis-producing cercaria of Austrobilharzia variglandis in California (Trematoda: Schistosomatidae). J Parasitol 44: 633-638, 1958.

28. Horák P, Kolářová L. Survival of bird schistosomes in mammalian lungs. Int J Parasitol 30: 65-68, 2000.

29. Horák P, Kolářová L. Bird schistosomes: do they die in mammalian skin? Trends Parasitol 17: 66-69, 2001.

30. Horák P, Kolářová L. Snails, waterfowl and cercarial dermatitis. Freshwater Biol 56: 779-790, 2011.

31. Horák P, Kolářová L, Adema CM. Biology of the schistosome genus Trichobilharzia. Adv Parasitol 52: $155-233,2002$.

32. Horák P, Mikes L, Rudolfová J, Kolářová L. Penetration of Trichobilharzia cercariae into mammals: dangerous or negligicle event? Parasite 15: 299-303, 2008.

33. Hunter GW, Ritchie LS, Tanabe H. The epidemiology of schistosome dermatitis ("koganbyo") in Japan. Trans R Soc Trop Med Hyg 45: 103-112, 1951.

34. Jouet D, Ferte H, Hologne C, Kaltenbach ML, Depaquit J. Avian schistosomes in French aquatic birds: a molecular approach. J Helminthol 83: 181-189, 2009.

35. Kohn A. Sôbre o gênero Macrobilharzia Travassos, 1922 (Trematoda, Schistosomatoidea). Mem Inst Oswaldo Cruz 62: 1-6, 1964.

36. Kolářová L. Schistosomes causing cercarial dermatitis: a mini-review of current trends in systematics and of host specificity and pathogenicity. Folia Parasitol 54: 81-87, 2007.

37. Kolářová L, Horák P, Skírnisson K. Methodical approaches in the identification of areas with a potential risk of infection by bird schistosomes causing cercarial dermatitis. $J$ Helminthol 84: 327-335, 2010.

38. Kolářová L, Horák P, Sitko J. Cercarial dermatitis in focus: schistosomes in the Czech Republic. Helminthologia 34: 127-139, 1997.

39. Kolářová L, Sýkora J, Bah BA. Serodiagnosis of cercarial dermatitis with antigens of Trichobilharzia szidati and Schistosoma mansoni. Cent Eur J Public Health 2: 19-22, 1994.

40. Kolářová L, Skírnisson K, Horák P. Schistosome cercariae as the causative agent of swimmer's itch in Iceland. J Helminthol 73: 215-220, 1999.

41. Kouřilová P, Kolářová L. Variations in immunofluorescent antibody response against Trichobilharzia and Schistosoma antigens in compatible and incompatible hosts. Parasitol Res 88: 513-521, 2002.

42. Kouřilová P, Hogg KG, Kolářová L, Mountford AP. Cercarial dermatitis caused by bird schistosomes comprises both immediate and late phase cutaneous hypersensitivity reactions. $J$ Immunol 172: 3766-3774, 2004a.

43. Kouřilová $\mathrm{P}$, Syrček M, Kolářová L. The severity of mouse pathologies caused by the bird schistosome Trichobilharzia regenti in relation to host immune status. Parasitol Res 93: 8-16, 2004b.

44. Kruatrachue M, Bhaibulaya M, Chesdapan C, Harinasuta C. Trichobilharzia maegraithi sp. nov., a cause of cercarial dermatitis in Thailand. Ann Trop Med Parasitol 62: 67-73, 1968.

45. La Valette de St. George AJH. Symbolae ad trematodum evolutionis historiam. Dissertation, Berolius, 1855.

46. Larsen AH, Bresciani J, Buchmann K. Increasing frequency of cercarial dermatitis in higher latitudes. Acta Parasitol 49: 217-221, 2004.

47. Leite ACR, Costa HMA, Costa JO. Trichobilharzia jequitibaensis n. sp. (Trematoda: Schistosomatidae) in Cairina moschata domestica (Anatidae). Rev Bras Biol 38: 843-846, 1978.

48. Leite ACR, Costa HMA, Costa JO. The life cycle of Trichobilharzia jequitibaensis Leite, Costa and Costa, 1978 (Trematoda, Schistosomatidae). Rev Bras Biol 39: 341-345, 1979.

49. Leite ACR, Costa HMA, Costa JO, Guimarães MP, Lima WS. The life cycle of Dendritobilharzia anatinarum Cheatum, 1941 (Trematoda, Schistosomatidae). Mem Inst Oswaldo Cruz 77: 389-396, 1982.

50. Li C, Ma C, Gu J, Lu W. An epidemiological survey of schistosome cercarial dermatitis among the residents living along the banks of the Huaihe River system. Chin J Parasitol Parasitic Dis 16: 384-387, 1998. 
51. Lichtenbergová L, Lassmann H, Jones MK, Kolářová L, Horák P. Trichobilharzia regenti: Host immune response in the pathogenesis of neuroinfection in mice. Exp Parasitol 128: 328-335, 2011.

52. Lindblade KA. The epidemiology of cercarial dermatitis and its association with limnological characteristics of a Northern Michigan Lake. J Parasitol 84: 19-23, 1998.

53. Liu Z, Chen M, Jin C, Tang Y, Yang F. A survey of the aetiological agent of addy-field dermatitis in Jian Xian, Jilin province, with preliminary observations of the life history of Trichibilharzia jianensis sp. nov. (Trematoda: Schistosomatidae). Acta Zool Sin 23: 161-174, 1977.

54. Lutz A. O Schistosoma mansoni e a schistosomatose segundo observações feitas no Brasil. Mem Inst Oswaldo Cruz 11: 121-155, 1919.

55. Lutz A. Notas sobre dicranocercarias brazileiras. Mem Inst Oswaldo Cruz 28: 349-376, 1933.

56. Macfarlane WV. Schistosome dermatitis in New Zealand: part I: the Parasite Am J Epidemiol 50: 143-151, 1949.

57. Macfarlane WV. Schistosome dermatitis in Australia. Med J Aust 39: 669-672, 1952.

58. Martin FS, Vicente FS. The life cycle of Trichobilharzia salmanticensis n. sp. (Digenea: Schistosomatidae), related to cases of human dermatitis. Res Rev Parasitol 59: 13-18, 1999.

59. Mas-Coma S, Valero MA, Bargues MD. Climate change effects on trematodiases, with emphasis on zoonotic fascioliasis and schistosomiasis. Vet Parasitol 163: 264-280, 2009.

60. Matheson S. Notes on Cercaria elvae Miller as the probable cause of an outbreak of dermatitis in Cardiff. Trans R Soc Trop Med Hyg 23: 421, 1930.

61. Meinking TL, Burkhart CN, Burkhart CG. Changing paradigms in parasitic infections: common dermatological helminthic infections and cutaneous myiasis. Clin Dermatol 21: 407-416, 2003.

62. Melo AL. Caracterização de larvas de trematódeos emergentes de moluscos dulciaquícolas. In: Amaral RS, Thiengo SC, Pieri OS, (org). Vigilância e controle de moluscos de importância epidemiológica: diretrizes técnicas: Programa de Vigilância e Controle da Esquistossomose (PCE). 2 ed. Ministério da Saúde. Brasília, 2008.

63. Mohr LV. Helmintofauna do marrecão, Netta peposaca (Vieillot, 1816) e da marreca-caneleira, Dendrocygna bicolor (Vieillot, 1816) no Rio Grande do Sul. Porto Alegre [Dissertação de Mestrado em Biologia Animal - UFRS], 2001.

64. Morley NJ. Cercarial dermatitis in the UK: a long established history. Clin Exp Dermatol 34: e443, 2009.

65. Neves J, Martins NR, Tonelli E. Forma toxêmica da esquistossomose mansoni. Considerações diagnósticas em torno de 50 casos identificados em Belo Horizonte. An Fac Med Minas Gerais 22: 75-98, 1965.

66. Neves J, Santiago JM, Ratton JLA. Quadro clínico. In: Cunha AS. Esquistossomose mansoni. Editora da USP. São Paulo, 1970.

67. Olivier L. Schistosome dermatitis, a sensitization phenomenon. Am J Hyg 49: 290-302, 1949.

68. Olivier L. Observations on the migration of avian schistosomes in mammals previously unexposed to cercariae. J Parasitol 39: 237-243, 1953.

69. Ostrowski de Núñez M. Fauna de agua dulce de la República Argentina, VII: cercarias de la familia Schistosomatidae (Trematoda: Digenea). Rev Mus Arg Cienc Nat Bernardino Rivadavia, Parasitol 2: 65-76, 1978.

70. Ostrowski de Núñez M. Trematoda. Familias Strigeidae, Diplostomidae, Clinostomidae, Schistosomatidae, Spirorchiidae y Bucephalidae. Fauna de Água Dulce de la República Argentina 9: $1-55,1992$.

71. Paulsen, RMM. Caracterização de helmintos e artrópodos parasitos de Netta peposaca (Vieillot, 1816) (Marrecão-da-patagônia) (Aves: Anatidae) no Rio Grande do Sul. Porto Alegre [Tese de Doutorado em Ciências Veterinárias - UFRS], 2006.

72. Penner LR. Cercaria littorinalinae sp. nov., a dermatitis-producing schistosome larva from the marine snail, Littorina planaxis Philippi. J Parasitol 36: 466-472, 1950.

73. Pessôa SB, Martins AV. Parasitologia Médica. 11ed. Guanabara Koogan. Rio de Janeiro, 1988.

74. Raso P, Bogliolo L. Patologia. In: Cunha AS. Esquistossomose mansoni. Editora da USP. São Paulo, 1970. 
75. Rizevsky SV, Cherviakovsky EM, Kurchenko VP. Molecular taxonomic identification of Schistosomatidae from Naroch Lake and Polonevichi Lake in Belarus. Biochem Syst Ecol 39: 14-21, 2011.

76. Schets FM, Lodder WJ, van Duynhoven YTHP, Husman AMR. Cercarial dermatitis in the Netherlands caused by Trichobilharzia spp. J Water Health 6: 187-195, 2008.

77. Skírnisson K, Aldhoun JA, Kolářová L. A review on swimmer's itch and the occurrence of bird schistosomes in Iceland. J Helminthol 83: 165-171, 2009.

78. Soleng A, Mehl R. Geographical distribution of cercarial dermatitis in Norway. J Helminthol 85: 345-352, 2011.

79. Souza FPC, Vitorino RR, Costa AP, Faria Jr FC, Santana LA, Gomes AP. Esquistossomose mansônica: aspectos gerais, imunologia, patogênese e história natural. Rev Bras Clin Med 9: 300-307, 2011.

80. Suzuki N, Kawanaka M. Trichobilharzia brevis Basch, 1966, as a cause of an outbreak of cercarial dermatitis in Japan. Jap J Parasitol 29: 1-11, 1980.

81. Stunkard HW, Hinchliffe MC. The morphology and life history of Microbilharzia variglandis (Miller and Northrup, 1926), Stunkard and Hinchliffe, 1951, avian blood-flukes whose larvae cause "swimmers-itch" of ocean beaches. J Parasitol 38: 248-265, 1952.

82. Szidat L. Cercarias schistosomicas y dermatitis schistosomica humana en la República Argentina. Commun Inst Nac Invest Cienc Nat 2: 129-150, 1951.

83. Szidat L, Szidat UC. Eine neue dermatitis erzeugende cercarie der trematoden-familie Schistosomatidae aus Tropicorbis peregrinus (d’Orbigny) des Río Quequen. Z Parasitenkd 20: 359-367, 1960.

84. Tanaka H, Tsuji M. From discovery to eradication of schistosomiasis in Japan: 1847-1996. Int J Parasitol 27: 1465-1480, 1997.

85. Thors C, Linder E. Swimmer's itch in Sweden. Helminthologia 38: 244, 2001.

86. Thors C, Linder E, Bylund G. Swimmers' itch. In: Akuffo H, Linder E, Ljungström I, Wahlgren M (eds). Parasites of the Colder Climates. Taylor \& Francis. London and New York, 2003.

87. Travassos L. Informações sobre a fauna helmintológica de Mato Grosso. Folha Med 3: 187-190, 1922.

88. Travassos L. Notas sobre trematódeos. An Acad Brasil Ciênc 4: 109, 1932.

89. Travassos L. Contribuições ao conhecimento dos Schistosomatidae. Sôbre Ornithobilharzia canaliculata (Rudolphi, 1819). Rev Bras Biol 2: 473-476, 1942.

90. Valdovinos C, Balboa C. Cercarial dermatitis and lake eutrophication in south-central Chile. Epidemiol Infect 136: 391-394, 2008.

91. Van den Broek E. Some recent cases of avian schistosomiasis and schistosome dermatitits in the Netherlands. Trop Geog Med 17: 229-235, 1965.

92. Verbrugge LM, Rainey JJ, Reimink RL, Blankespoor HD. Swimmer's itch: incidence and risk factors. Am J Public Health 94: 738-741, 2004.

93. Wulff C, Haeberlein S, Haas W. Cream formulations protecting against cercarial dermatitis by Trichobilharzia. Parasitol Res 101: 91-97, 2007.

94. Zbikowska E. Infection of snails with bird schistosomes and the threat of swimmers itch in selected Polish lakes. Parasitol Res 92: 30-35, 2004. 